\title{
Adaptation to Low-Intensity Exercise on a Cycle Ergometer by Patients With Acute Myocardial Infarction Undergoing Phase I Cardiac Rehabilitation
}

\author{
Atsuhiko Matsunaga, PhD; Takashi Masuda, MD; Misao N Ogura, MS; \\ Masakazu Saitoh, MS*; Yusuke Kasahara, MS*; Takami Iwamura, BA**; \\ Minako Yamaoka-Tojo, $\mathrm{MD}^{\dagger}$; Kiyotaka Sato, $\mathrm{MD}^{\dagger}$; Tohru Izumi, $\mathrm{MD}^{\dagger}$
}

\begin{abstract}
Background The adaptation of patients with acute myocardial infarction (AMI) to a phase I rehabilitation program has not been widely assessed.

Methods and Results Forty-two male patients (62 \pm 8 years) with AMI were classified as exercise tolerant (group $\mathrm{A}, \mathrm{n}=25$ ) or excessive response (systolic blood pressure (SBP) increase $>30 \mathrm{mmHg}$ during exercise; group $\mathrm{B}, \mathrm{n}=17$ ). Hemodynamic parameters during exercise using a cycle-ergometer were monitored for the first 3 days. The power of low- and high-frequency components (LF: $0.05-0.2 \mathrm{~Hz}$; HF: $0.2-1 \mathrm{~Hz}$ ) was analyzed by heart rate variability. Anxiety status was assessed using the Spielberger's State-Trait Anxiety Inventory. Patients in group B were significantly older, had lower cardiac function and a longer hospitalization than group A $(\mathrm{p}<0.05$, respectively). The excessive elevation of SBP on Day 1 decreased and became $<30 \mathrm{mmHg}$ on Day 3 in group B. The decreases in HF during exercise on Days 1 and 3 were significantly smaller in group B than in group $A(p<0.05$ and $p<0.05$, respectively). The LF/HF ratio on Day 1 was significantly higher in group $B$ than in group A $(\mathrm{p}<0.05)$. In group $\mathrm{B}$, the anxiety score before exercise was significantly higher than that at the time of discharge $(\mathrm{p}<0.05)$, whereas there was no change in group A.
\end{abstract}

Conclusion Factors influencing a significant elevation of blood pressure during phase I rehabilitation are age, physical deconditioning, imbalance of autonomic nervous activity and anxiety. (Circ J 2004; 68: 938-945)

Key Words: Acute myocardial infarction; Anxiety; Autonomic nervous activity; Ergometer; Therapeutic exercise

$\mathbf{I}$ $\mathrm{t}$ is now common for patients with acute myocardial infarction (AMI) to undergo exercise testing using a treadmill or cycle ergometer, the most common equipment used in clinical practice for exercise testing and training,-4 during their hospital stay (phase I) to assess the post-hospital physical activity, medical treatment and prognosis:-12 Generally, aerobic exercise training lasting up to 30 min using a cycle ergometer or treadmill is carried out prior to hospital discharge, following the resumption of basic activities such as sitting, standing, self-care and level walking within the ward in the early days after AMI!3-17 This predischarge exercise training can provide information on cardiovascular response that is useful for setting home activities guidelines, outpatient programs and socializing such as return to work after hospital discharge!2-14

When exercise training using equipment is prescribed, the training equipment should be chosen after due consideration of its suitability for the patient's physical condition.

(Received March 9, 2004; revised manuscript received July 12, 2004; accepted July 29, 2004)

Department of Rehabilitation, School of Allied Health Sciences, *Graduate School of Medical Sciences, Kitasato University, **Rehabilitation Center, Kitasato University Hospital and Department of Internal Medicine, School of Medicine, Kitasato University, Kanagawa, Japan

Mailing address: Takashi Masuda, MD, PhD, Professor, Department of Rehabilitation, School of Allied Health Sciences, Kitasato University, 1-15-1 Kitasato, Sagamihara, Kanagawa 228-8555, Japan.

E-mail: tak9999@med.kitasato-u.ac.jp
In particular, the stationary cycle ergometer seems to be an excellent training equipment for post-MI patients whose heart rate (HR), blood pressure (BP) and electrocardiogram (ECG) have to be fully monitored during a phase I cardiac rehabilitation program 3,18 The ergometer is often used for patients who have orthopedic and neurological problems, including muscle weakness and gait disturbance, because it enables not only weight-bearing but also nonweightbearing training because workload is easily adjusted!,18 Furthermore, several studies have reported comparisons of the cardiovascular response to submaximal exercise with a cycle ergometer versus a treadmill. Although maximal exercise with a cycle ergometer produced higher BP and lower oxygen uptake than on a treadmill, cardiovascular response and oxygen uptake during cycle ergometer exercise at $40 \mathrm{~W}$, corresponding to 3.5 metabolic equivalents or less than anaerobic intensity level, were close to those during equivalent treadmill exercise ${ }^{19-22}$

However, there are few studies demonstrating the adaptation of post-MI patients to exercise training using either a treadmill or cycle ergometer, although exercise testing is reportedly useful in terms of safety, efficacy and clinical benefit in the early days after AMI23,24 We have had patients who showed excessive elevation of BP or HR during exercise training as part of phase I cardiac rehabilitation, without other symptoms or signs related to myocardial ischemia 25 If the clinical characteristics on admission affect the patient's ability to adapt to exercise training using a cycle ergometer in the early days after AMI, it is very im- 


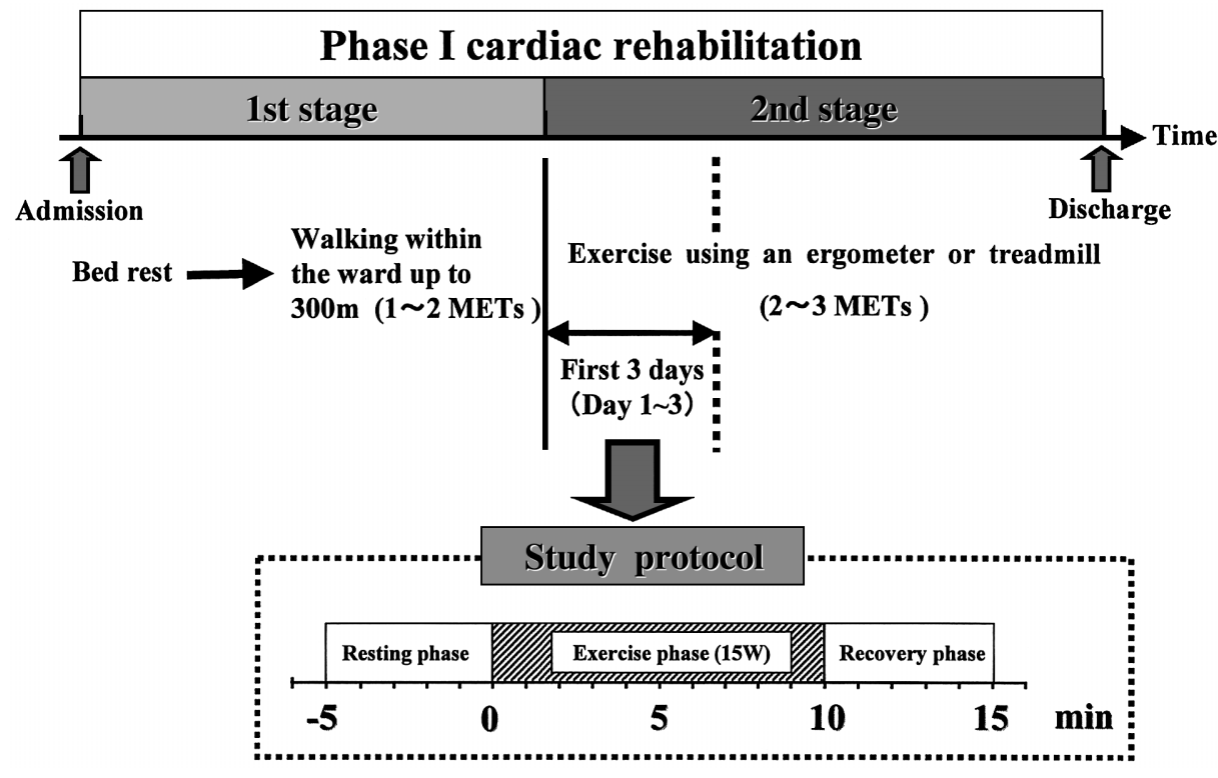

Fig 1. Phase I cardiac rehabilitation program and study protocol. METs, metabolic equivalents; W, watts.

portant to evaluate these factors and then clinicians can safely prescribe an individual exercise program suitable with regard to the intensity, duration and frequency of the exercise.

In the present study, we correlated clinical manifestations with changes in HR, BP and autonomic nervous activity during low-intensity exercise using a cycle ergometer in post-MI patients to clarify their adaptation to exercise training during phase I cardiac rehabilitation.

\section{Methods}

\section{Subjects}

The study protocol was approved by the Ethics Committee of Kitasato University Hospital, and informed consent was obtained from all patients after detailed explanation of the study protocol. Patients who were admitted to the Cardiovascular Center of Kitasato University Hospital because of AMI were eligible for this study if they underwent percutaneous coronary intervention following coronary angiography (CAG) to detect the coronary lesion, and then could walk up to $300 \mathrm{~m}$ within the ward without developing any symptoms or significant ECG changes. Exclusion criteria were cardiac decompensation, arrhythmias including atrial fibrillation and frequent ventricular extrasystole, pacemaker implantation, chronic renal failure on hemodialysis, diabetes mellitus, chronic obstructive pulmonary disease and noncardiac conditions limiting exercise performance, such as orthopedic abnormalities and stroke. Consequently, 42 male patients with AMI (mean age: $62 \pm 8$ years) were enrolled for the present study.

AMI was documented by the combination of anamnesis, elevation of serum creatine kinase (CK) and the MB isoenzyme of CK (CK-MB), and characteristic ST-T changes or the presence of a new abnormal Q wave on the ECG.

The cardiac rehabilitation program supervised in the Cardiovascular Center consists of 2 exercise stages (Fig 1). The first stage is basic activities, such as sitting up in bed, sit-to-stand, self-care and walking in the ward, that are usually started the second day after AMI. After the patients complete the first stage, they proceed to the second stage:
Table 1 Criteria for Phase I Cardiac Rehabilitation Recommended by the Research Committee of the Japanese Ministry of Health and Welfare

1 Absence of angina on exertion, palpitations, dyspnea, fatigue and dizziness.

2 Absence of $>1 \mathrm{~mm}$ ST segment depression, or $>2 \mathrm{~mm}$ ST segment elevation on the ECG.

3 Absence of significant (uncontrolled) atrial or ventricular arrhythmia.

4 Heart rate $<120$ beats $/ \mathrm{min}$.

5 Increase in systolic blood pressure $<30 \mathrm{mmHg}$.

6 Decrease in systolic blood pressure $<20 \mathrm{mmHg}$.

aerobic training using a cycle ergometer or treadmill in the Cardiac Rehabilitation Center.

\section{Study Protocol}

The study was carried out during 3 days at the beginning of the second stage (Fig 1). We classified the patients as exercise tolerant (group A) or excessive response (group B), according to the criteria for a phase I cardiac rehabilitation program recommended by the Research Committee of the Japanese Ministry of Health and Welfare (Table 1)25 The evaluation, which consisted of 3 phases, was performed once each day and was repeated for the 2 groups during the first 3 days after entering the second stage. After sitting on an electronically braked cycle ergometer (Well Bike BE360, Fukuda Denshi, Tokyo, Japan) for $5 \mathrm{~min}$ (resting phase), the patients had to exercise in the upright posture for $10 \mathrm{~min}$ at a low-intensity workload of $15 \mathrm{~W}$ and a pedal frequency of $50 \mathrm{rpm}$ (exercise phase), after which they rested again while sitting on the ergometer for $5 \mathrm{~min}$ (recovery phase).

In this study protocol all patients were directed to rest, without performing any other exercise such as walking within the ward, for at least $1 \mathrm{~h}$ before beginning the cycle ergometer training. To assess the cardiovascular response to aerobic training using the ergometer, the HR and ECG changes, including arrhythmia, were continuously monitored using a Stress Test System (ML-1800, Fukuda 
Table 2 Clinical Characteristics of the AMI Patients in Groups A and B

\begin{tabular}{lcc}
\hline \hline & Group A & Group B \\
\hline No. of patients & $25(59.5 \%)$ & $17(40.5 \%)$ \\
Age (years) & $57.1 \pm 1.7$ & $68.6 \pm 2.4^{\dagger}$ \\
Height $(\mathrm{cm})$ & $163.8 \pm 1.8$ & $160.2 \pm 2.1$ \\
Weight $(\mathrm{kg})$ & $57.5 \pm 2.3$ & $56.2 \pm 2.4$ \\
Body mass index & $21.8 \pm 0.6$ & $22.2 \pm 0.8$ \\
No. of patients with significant coronary artery stenosis (>75\%) before PCI & & $*$ \\
SVD & 10 & 5 \\
DVD & 5 & 2 \\
TVD & 0 & 5 \\
Infarct-related artery (no. of patients) & & 4 \\
Right coronary artery & 5 & 6 \\
Left anterior descending artery & 8 & 1 \\
Left circumflex artery & 2 & $40.1 \pm 2.3^{*}$ \\
Left ventricular ejection fraction (\%) & $52.3 \pm 2.0$ & $3,650 \pm 377^{*}$ \\
Peak CK (IU/L) & $2,280 \pm 205$ & $350 \pm 39^{*}$ \\
Peak CK-MB (IU/L) & $240 \pm 20$ & \\
Duration of hospitalization from admission to start of rehabilitation (days) & & $12.4 \pm 1.7^{*}$ \\
Level walking within the ward $(100 \mathrm{~m})$ & $8.6 \pm 0.9$ & $16.8 \pm 1.4^{*}$ \\
Level walking within the ward $(300 \mathrm{~m})$ & $12.8 \pm 0.9$ & 10 \\
Medication (no. of patients) & & 2 \\
Nitrate & 13 & 9 \\
Calcium channel antagonist & 3 & 10 \\
B -blocker & 12 & \\
ACE inhibitor & 12 & \\
\hline
\end{tabular}

Data are mean $\pm S E$.

$A C E$, angiotensin-converting enzyme; AMI, acute myocardial infarction; PCI, percutaneous coronary intervention; SVD, single vessel disease; $D V D$, double vessel disease; TVD, triple vessel disease.

* and ${ }^{\dagger} p<0.05$ and $p<0.01$ vs group $A$, respectively.

Denshi), and BP was measured each minute by the cuff method using an automatic manometer (FB-300, Fukuda Denshi) throughout the 3 phases. To analyze the heart rate variability (HRV), Holter ECG recording in the CM5, NASA and aVF leads (FM-300, Fukuda Denshi) was performed twice on Days 1 and 3, from $1 \mathrm{~h}$ before the exercise training with cycle ergometer until after its termination. All patients were instructed to interrupt the exercise if they experienced adverse symptoms such as chest discomfort, shortness of breath and muscular fatigue.

\section{Data Analysis}

Clinical Characteristics Age, body weight, height and body mass index (BMI) were recorded just before entering the study. The infarct-related artery and the number of coronary arteries showing significant stenosis $(>75 \%)$ before revascularization were determined by emergency $\mathrm{CAG}$ on admission. The serum concentration of CK and CK-MB was measured every $6 \mathrm{~h}$ after admission, and then the peak concentration of each was decided. Left ventricular ejection fraction (LVEF) was evaluated based on the left ventriculogram performed on admission. The periods from the first hospital day to the days the patient could walk $100 \mathrm{~m}$ and $300 \mathrm{~m}$ were recorded in the first stage. Most patients were treated with nitrates, calcium channel antagonists, $\beta$-blockers or angiotensin-converting enzyme inhibitors after admission and their medication was not changed throughout the study to avoid any influence on the cardiovascular response during the exercise.

Power Spectral Analysis of HRV The HRV obtained from the Holter ECG was analyzed by wavelet transformation to evaluate autonomic nervous activity ${ }^{26}$ The sampling rate was $1,000 \mathrm{~Hz}$, thus giving $1-\mathrm{ms}$ time resolution for the $\mathrm{RR}$ interval. $\mathrm{R}$ wave detection and visual inspection of the $\mathrm{RR}$ intervals were performed by the operator, before the export of consecutive RR intervals to a digital data file for spectral analysis of HRV. Premature supraventricular and ventricular beats, missed beats and pauses that could affect the result of spectral analysis were filtered and replaced by an interpolated value according to a cubic spline function curve fitting? 27,28 The spectra of HRV were expressed as low-frequency (LF: 0.05-0.2 Hz) and high-frequency (HF: $0.2-1.0 \mathrm{~Hz})$ components. The power $\left(\mathrm{ms}^{2}\right)$ of $\mathrm{LF}$ and $\mathrm{HF}$ was calculated at 1-min intervals, and the $\mathrm{HF}$ and LF/HF ratio were used as indexes of parasympathetic and sympathetic nervous activities, respectively.

Changes in the HR, SBP, HF and LF/HF Ratio We defined the values of HR, systolic blood pressure (SBP), HF and $\mathrm{LF} / \mathrm{HF}$ ratio obtained $3 \mathrm{~min}$ after the beginning of the resting phase as the baseline values for each day. The HR5 min and HR10min, SBP5 min and SBP10min, HF5 min and HF10 min, and LF/HF5 min and LF/HF10 min indicated the values obtained $5 \mathrm{~min}$ and $10 \mathrm{~min}$, respectively, after the beginning of the exercise phase. To compare the changes in HR and SBP, the $\triangle \mathrm{HR}$ and $\triangle \mathrm{SBP}$, which indicated the differences from the baseline values to the HR5 min and SBP5 min ( $\triangle$ HR5 min and $\triangle$ SBP5 min, respectively) or to the HR10 min and SBP10 min $(\Delta \mathrm{HR} 10 \mathrm{~min}$ and $\triangle \mathrm{SBP} 10 \mathrm{~min}$, respectively), were calculated each time.

Anxiety Status The anxiety status was assessed twice, at the end of the first and second stages, using the Spielberger's State-Trait Anxiety Inventory (STAI) to clarify the effect of aerobic training on the patient's psychosocial condition? 29,30

The STAI is a self-reported assessment designed to measure anxiety, and comprises 2 separate scales: state and trait anxiety, 29,30 The state-anxiety scale, consisting of 20 statements, evaluates the temporary conditions of apprehension, tension, nervousness and worry, which vary in response to physical danger and psychological stress, 

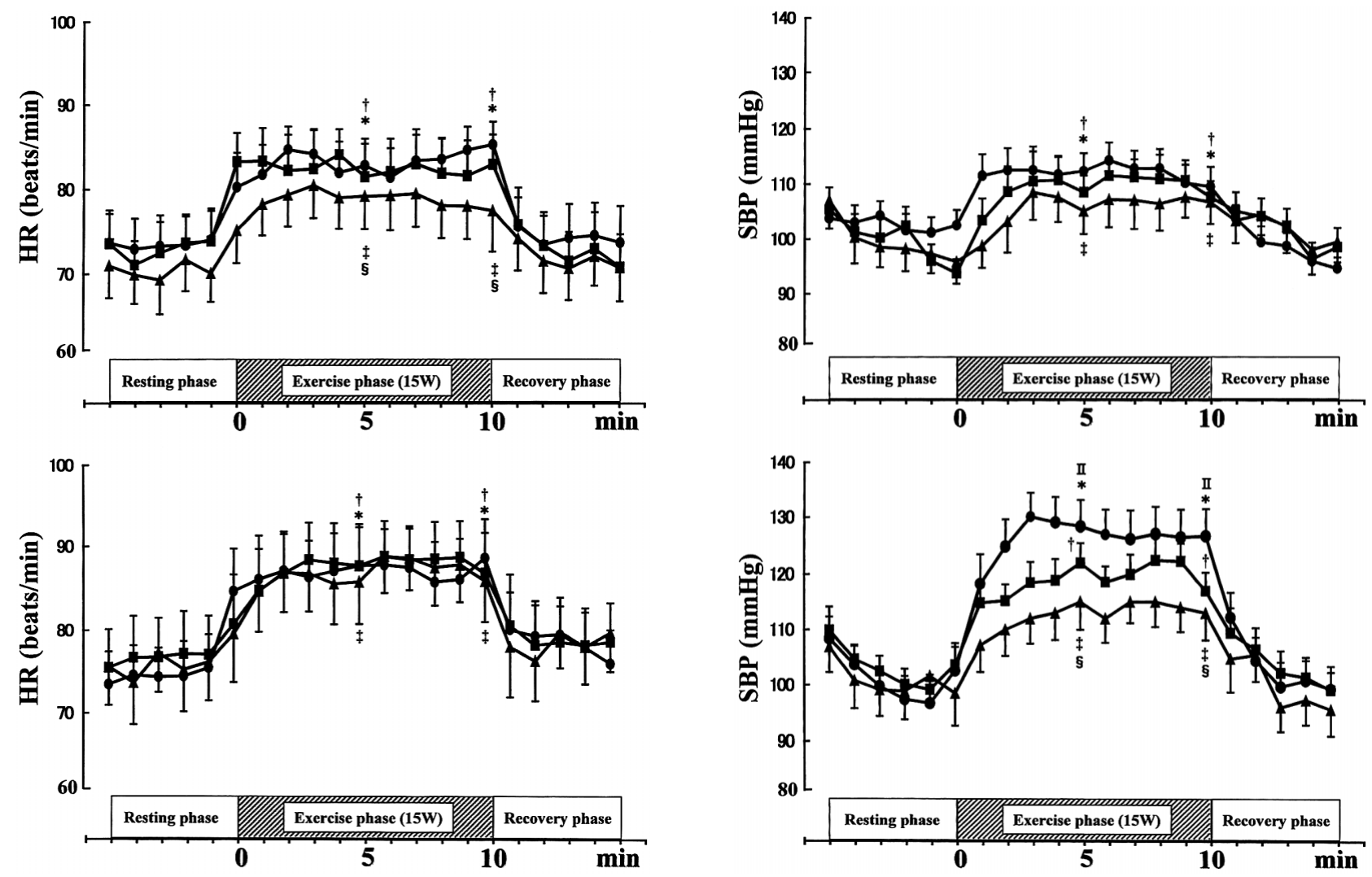

Fig 2. Time course of heart rate (HR) on Days 1-3 of the study in group A (Top) and B (Bottom). Data are mean \pm SE. Closed circles, closed squares and closed triangles indicate HR measured on Days 1, 2 and 3 of the study, respectively. ${ }^{*} \mathrm{p}<0.05$ vs baseline value measured on Day $1 ; \uparrow \mathrm{p}<0.05$ vs baseline value on Day $2 ;{ }^{\ddagger} \mathrm{p}<0.05$ vs baseline value on Day $3 ; s_{\mathrm{p}}<0.05$ between values measured on Day 1 and those measured on Day 3.

whereas the trait-anxiety scale, also consisting of 20 statements, evaluates continuous and uniform anxiety about the perceived threat in the environment. The lower the total score of the 2 scales, which ranges from 20 to 80 , the better the psychological status.

Statistical Analysis All values are expressed as the mean \pm standard error (SE) and differences in the clinical characteristics between groups A and B were assessed for statistical significance by Student's unpaired t-test and chisquare test. The changes in HR, SBP, HF and LF/HF ratio and STAI scores were analyzed by ANOVA with repeated measurements in each group. Differences were considered statistically significant if the $\mathrm{p}$ value was less than 0.05 .

\section{Results}

The clinical characteristics of the patients in groups A and $\mathrm{B}$ are summarized in Table 2 . In the present study 17 patients who belonged to group B withdrew because they experienced an increase in SBP $>30 \mathrm{mmHg}$ during exercise on Day 1 . The mean age of the group and the number of patients with triple vessel disease were significantly higher in group B than in group A ( $\mathrm{p}<0.01$ and $\mathrm{p}<0.05$, respectively). However, there were no significant differences in the distribution of the infarct-related arteries between the 2 groups. Group B showed significantly higher values of peak CK
Fig 3. Time course of systolic blood pressure (SBP) on Days 1-3 of the study in group A (Top) and B (Bottom). Data are mean \pm SE. Closed circles, closed squares and closed triangles indicate SBP measured on Days 1, 2 and 3 of the study, respectively. ${ }^{*} p<0.05$ vs baseline value measured on Day $1 ; \mathrm{p}<0.05$ vs baseline value on Day 2; $\ddagger_{\mathrm{p}}<0.05$ vs baseline value on Day $3 ; \S_{\mathrm{p}}<0.05$ between values measured on Day 1 and Day 3; ${ }^{\mathrm{I}} \mathrm{p}<0.05$ vs value measured on Day 1 in group $\mathrm{A}$.

and CK-MB ( $<<0.05$ and $\mathrm{p}<0.05$, respectively), lower LVEF $(\mathrm{p}<0.05)$ and a longer hospitalization period prior to $100-\mathrm{m}$ and $300-\mathrm{m}$ walking $(\mathrm{p}<0.05$ and $\mathrm{p}<0.05$, respectively) as compared with group A. No significant differences were shown in HR and BP during the exercise in the first stage between the 2 groups. There was no significant difference regarding the medication prescribed just before entering the second stage between the 2 groups.

Fig 2 shows the time course of HR measured on Days $1-3$ of the study in the 2 groups. Both HR 5 min and HR10 min increased significantly from the baseline values each day in the 2 groups $(\mathrm{p}<0.05$ and $\mathrm{p}<0.05$, respectively), but all values obtained during the exercise phase were less than 120 beats/min in the 2 groups. There were no significant differences in HR5 min or HR 10 min among the 3 days in group B, although those values decreased significantly on Day 3 as compared with those on Day 1 in group A $(\mathrm{p}<0.05$ and $\mathrm{p}<0.05$, respectively). The $\Delta$ HR5 min and $\Delta$ HR10 min showed no significant changes among the 3 days in either group, and no significant differences between the 2 groups. The HR decreased immediately after the exercise phase and returned to the baseline values at the recovery phase in each of the 3 days in the 2 groups.

Fig 3 shows the time course of SBP measured on Days 1-3 of the study in the 2 groups. Both SBP5 min and SBP10 min increased significantly from the baseline values 

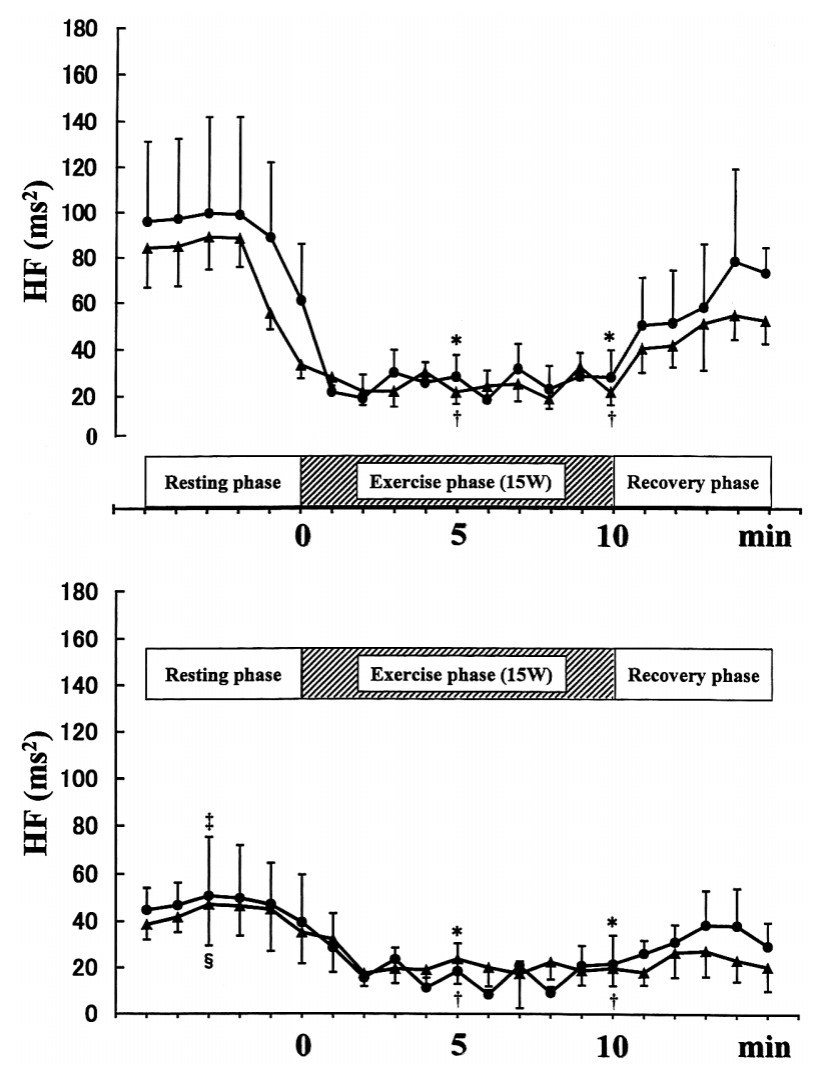

Fig 4. Time course of the high-frequency (HF) component on Days 1 and 3 of the study in group A (Top) and B (Bottom). Data are mean \pm SE. Closed circles and closed triangles indicate HF component measured on Days 1 and 3 of the study, respectively. ${ }^{*} \mathrm{p}<0.05$ vs baseline value measured on Day $1 ; \uparrow p<0.05$ vs baseline value on Day $3 ; \star \mathrm{p}<0.05$ vs value obtained on Day 1 in group $\mathrm{A} ;{ }^{\mathrm{p}}<0.05$ vs value obtained on Day 3 in group A.

each day in the 2 groups ( $\mathrm{p}<0.05$ and $\mathrm{p}<0.05$, respectively). Although there were no significant differences in SBP5 min and SBP10 min among the 3 days in group A, those values decreased significantly on Day 3 as compared with those of Day 1 in group $B(p<0.05$ and $\mathrm{p}<0.05$, respectively). The $\triangle \mathrm{SBP} 5 \mathrm{~min}$ and $\triangle \mathrm{SBP} 10 \mathrm{~min}$ on Day 1 were significantly higher in group $B$ than in group A $(p<0.05$ and $p<0.05$, respectively). The $\triangle \mathrm{SBP} 5 \mathrm{~min}$ and $\triangle \mathrm{SBP} 10 \mathrm{~min}$, which were $>30 \mathrm{mmHg}$ on Day 1, decreased gradually and were $<30 \mathrm{mmHg}$ on Day 3 in group B $(\mathrm{p}<0.05$ and $\mathrm{p}<0.05$, respectively). The SBP decreased immediately after the exercise phase and returned to the baseline values in the recovery phase each day in the 2 groups.

Fig 4 shows the time course of the HF component measured on Days 1 and 3 of the study in the 2 groups. In both groups, the HF5 min and HF10 min decreased significantly as compared with the baseline values on Days 1 and 3 ( $\mathrm{p}<$ 0.05 and $\mathrm{p}<0.05$, respectively), and then returned to the baseline values $3-5 \mathrm{~min}$ after the exercise phase. However, there were no significant differences between the HF measured on Day 1 and that of Day 3 in any phase in either group. The baseline values of HF on both Day 1 and Day 3 were significantly lower in group B than in group A ( $<<$ 0.05 and $\mathrm{p}<0.05$, respectively), although there were no significant differences in the HF5 min and HF10 min between the 2 groups.

Fig 5 shows the time course of the LF/HF ratio on Days
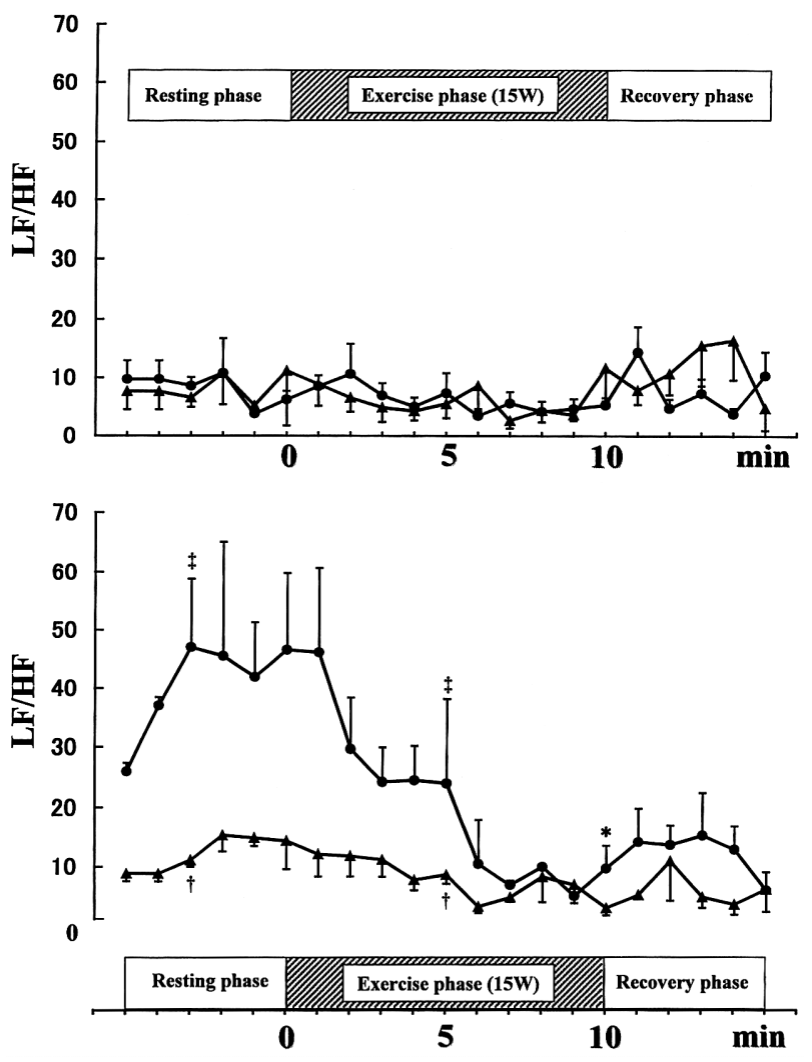

Fig 5. Time course of the LF/HF ratio on Days 1 and 3 of the study in group A (Top) and B (Bottom). Data are mean \pm SE. Closed circles and closed triangles indicate LF/HF ratio measured on Days 1 and 3 of the study, respectively. ${ }^{*} \mathrm{p}<0.05$ vs baseline value measured on Day $1 ;{ }^{\dagger} \mathrm{p}<0.05$ between the values on Days 1 and $3 ; \dot{\mathrm{p}}<0.05$ vs value obtained on Day 1 in group A. HF and LF, high- and low-frequency components.

1 and 3 of the study in the 2 groups. The LF/HF ratio in group A showed no significant changes either on Day 1 or on Day 3, and nor significant differences between the $\mathrm{LF} / \mathrm{HF}$ ratio obtained on Day 1 and that of Day 3. In group $\mathrm{B}$, the baseline $\mathrm{LF} / \mathrm{HF}$ ratio and $\mathrm{LF} / \mathrm{HF} 5$ min ratio were significantly higher on Day 1 as compared with Day 3 ( $\mathrm{p}<$ 0.05 and $\mathrm{p}<0.05$, respectively). The elevated $\mathrm{LF} / \mathrm{HF}$ ratio decreased gradually during the exercise phase and then the $\mathrm{LF} / \mathrm{HF} 10 \mathrm{~min}$ ratio became equal to the one obtained on Day 3. The baseline LF/HF ratio and LF/HF5 min ratio were significantly higher in group B than in group A on Day $1(\mathrm{p}<0.05$ and $\mathrm{p}<0.05$, respectively), but there were no significant differences in the LF/HF ratio obtained at any phase on Day 3 between the 2 groups.

Fig 6 shows the state- and trait-anxiety scores at the end of the first and second stages. The state-anxiety score assessed at the second stage was significantly lower than that measured at the first stage in group $B(p<0.05)$; there was no significant difference between them in group A. The state-anxiety score at the end of the second stage was significantly lower in group B than in group A $(\mathrm{p}<0.05)$, although there was no significant difference between the 2 groups at the end of the first stage. As for the trait-anxiety scores, there was no significant difference in either group between the first and second stages. Furthermore, there was no significant difference either in the trait-anxiety score assessed at the end of the first or the second stage between 
the 2 groups.

\section{Discussion}

Advances in medical intervention and primary care have contributed to reduce the mortality rate in the acute phase of MI, particularly during hospitalization. This has led to an increase in the number of patients with AMI who are further advanced in years and have lower cardiac function or multiple coronary risk factors. Therefore, careful consideration is needed regarding the prescription of exercise training in the phase I cardiac rehabilitation program for post-MI patients 31 Some researchers have focused on a phase II rehabilitation program (ie, supervised exercise training after hospital discharge) because the activities of daily living (ADL) of outpatients are restricted by the complications accompanying AMI! ${ }^{7}$ It is also known that the rate of adherence to rehabilitation after discharge affects infarct-related morbidity, including re-stenosis of coronary arteries and post-infarction angina ${ }^{32-34}$ To switch the rehabilitation program smoothly and safely from phase I to phase II, during hospitalization the patients should perform individually planned exercises directed toward the rehabilitation goal based on the ADL they would expect to perform after discharge.

For AMI patients complicated by noncardiac conditions that limit exercise performance, such as orthopedic, peripheral vascular or neurological disorders, a cycle ergometer is more useful than a treadmill because the exercise training can be conducted more safely while adjusting the workload incrementally2,4 Furthermore, the cardiovascular response during cycle ergometer exercise at submaximal exercise level is close to that obtained during equivalent treadmill exercise ${ }^{19-22}$ It must also be taken into account that patients have to learn how to use the equipment so that they can continue the exercise training at home or use facilities for sport and recreation after discharge 4

In the present study $40 \%$ (17/42) of the patients with AMI withdrew from the phase I rehabilitation program because the $\triangle \mathrm{SBP}$ was $>30 \mathrm{mmHg}$ during exercise, even at the low-intensity workload. These patients were older and had higher values of peak CK and peak CK-MB, and lower LVEF, and the period from onset of AMI to the beginning of walking was longer, as compared with group A. The patients in group B had severe cardiac dysfunction that delayed rehabilitation and resulted in physical deconditioning in the first stage. It has been suggested that major factors disturbing the progress of phase I rehabilitation are advanced age and deconditioning associated with cardiac dysfunction, $17,25,35$ but the present study demonstrated that the excessive increase of SBP observed on Day 1 of exercise decreased gradually and then returned to levels within the limits defined by the criteria for cardiac rehabilitation on Day 3 in group B. It is hard to believe that the reduction in $\triangle \mathrm{SBP}$ resulted from an amelioration of deconditioning, because low-intensity exercise using a cycle ergometer could not have strengthened the weak muscles of the extremities in those patients in just 3 days. Rather, the excessive increase of SBP may have been caused by their strained and excited mental state on Day 1. SBP gradually normalized by Day 3, probably because they became mentally adapted to exercise training using the cycle ergometer.

It is well recognized that the response of $\mathrm{HR}$ and BP to exercise training reflects changes in autonomic nervous system activity $3^{6-42}$ To assess these effects on the elevation
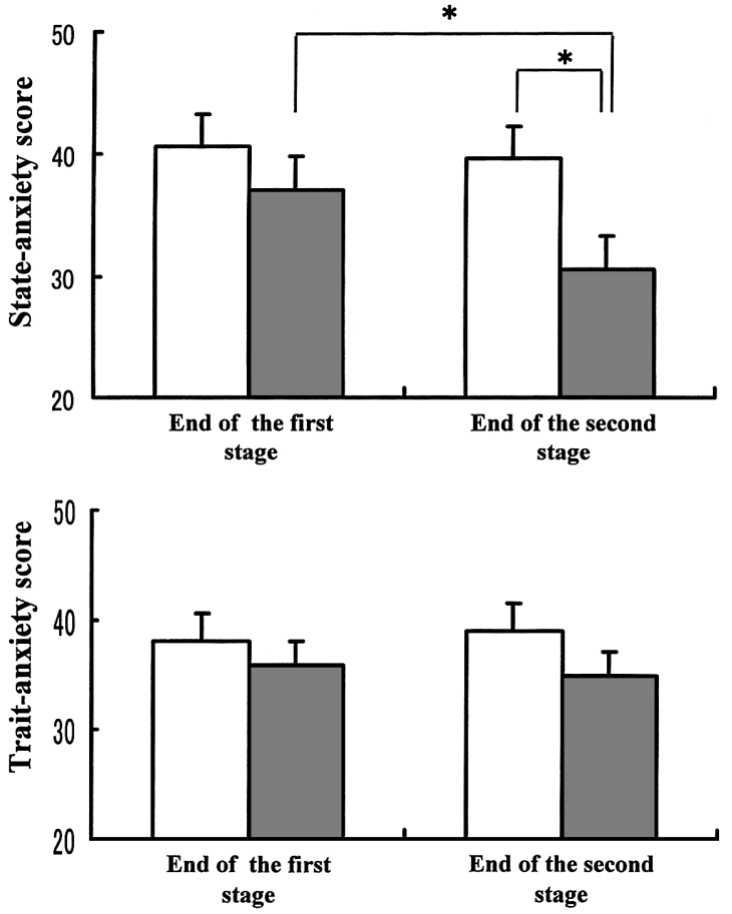

Fig 6. State-anxiety (Top) and trait-anxiety (Bottom) scores assessed at the end of the first and second stages. Each column and bar represent the mean value \pm SE. Open and closed columns indicate group A and $\mathrm{B}$, respectively. ${ }^{*} \mathrm{p}<0.05$.

of SBP during exercise training, we analyzed HRV using wavelet transformation and compared the sympathetic and parasympathetic activities. The marked activation of the sympathetic nervous system by high-intensity exercise is known to contribute to the increased HR and the augmentation of systemic vascular resistance results in the elevation of BP $3^{3}$ It has also been reported that reduced parasympathetic nervous activity participates in the increased HR at low-intensity exercise in healthy subjects.43,44 The present study demonstrated that the increase in HR could be mainly attributed to decreased parasympathetic nervous activity in group A, and predominantly to increased sympathetic nervous activity with a smaller decrease in parasympathetic nervous activity in group B.

The intensity of the exercise performed in the present study seemed to be relatively higher in group B than in group A, because the patients in group B were older and had deconditioning associated with cardiac dysfunction. This might have resulted in the marked elevation of sympathetic nervous activity in group B as compared with group A. There are many reports documenting that sympathetic nervous activity increases to up-regulate cardiac dysfunction in patients with heart failure,4-47 although parasympathetic nervous activity generally predominates during the resting state in healthy humans 43,44 The results of the present study support those reports because a marked increase in sympathetic nervous activity was observed during lowintensity exercise in group $\mathrm{B}$, in which many patients had a lower LVEF indicative of left ventricular dysfunction.

One of the reasons for the marked increase in sympathetic nervous activity observed on Day 1 in group B is considered to be that the more aged persons were more anxious because of their inexperience with the exercise modality; that is, the cycle ergometer ${ }^{17,48,49}$ Indeed, the 
state-anxiety score decreased significantly after training in group B only and anxiety about the exercise training on Day 1 resulted in the increase in the LF/HF ratio even during the resting phase in group B. However, the present study showed that the state-anxiety score at the end of the second stage was higher in group A than in group B, which might reflect their anxiety just before hospital discharge about their psychosocial status, such as reinstatement, which would be unrelated to the exercise training, because group A consisted of younger patients who would return to work after discharge.

We conclude that the factors influencing withdrawal from a phase I rehabilitation program using a cycle ergometer are age, deconditioning associated with cardiac dysfunction, and anxiety because they lead to an increase in sympathetic nervous activity. In addition, the instructors conducting cardiac rehabilitation need to explain the exercise procedure sufficiently to the patients before the training and carefully observe their cardiovascular responses, even with low-intensity exercise, for the first several days.

\section{Acknowledgement}

This study was supported in part by a grant-in-Aid for scientific research (No.15500383) from the Ministry of Education, Science, and Culture in Japan.

\section{References}

1. American College of Sports Medicine. Clinical exercise testing. In: ACSM's guidelines for exercise testing and prescription, 6th edn. Philadelphia: Lippincott Williams \& Wilkins; 2000; 91-114.

2. Fletcher GF, Balady GJ, Amsterdam EA, Chaitman B, Eckel R, Fleg $\mathrm{J}$, et al. Exercise standards for testing and training: A statement for healthcare professionals from the American Heart Association. Circulation 2001; 104: 1694-1740.

3. Fardy PS, Yanowitz FG, Wilson PK. Cardiac rehablitation, adult fitness, and exercise testing, 3rd edn. Philadelphia: Lippincott Williams \& Wilkins; 1996; 55-278.

4. Pina IL, Balady GJ, Hanson P, Labovitz AJ, Madonna DW, Myers J. Guidelines for clinical exercise testing laboratories: A statement for healthcare professionals from the Committee on Exercise and Cardiac Rehabilitation, American Heart Association. Circulation 1995; 91: 912-921.

5. Fuller CM, Raizner AE, Verani MS, Nahormek PA, Chahine RA, McEntee CW, et al. Early post-myocardial infarction treadmill stress testing: An accurate predictor of multivessel coronary disease and subsequent cardiac events. Ann Intern Med 1981; 94: 734-739.

6. Weld FM, Chu KL, Bigger JT Jr, Rolnitzky LM. Risk stratification with low-level exercise testing 2 weeks after acute myocardial infarction. Circulation 1981; 64: 306-314.

7. Birk Madsen E, Gilpin E, Ahnve S, Henning H, Ross J Jr. Prediction of functional capacity and use of exercise testing for predicting risk after acute myocardial infarction. Am J Cardiol 1985; 56: 839-845.

8. Ciaroni S, Delonca J, Righetti A. Early exercise testing after acute myocardial infarction in the elderly: Clinical evaluation and prognostic significance. Am Heart J 1993; 126: 304-311.

9. Krone RJ, Gillespie JA, Weld FM, Miller JP, Moss AJ. Low-level exercise testing after myocardial infarction: Usefulness in enhancing clinical risk stratification. Circulation 1985; 71: 80-89.

10. American College of Sports Medicine. Physical fitness testing and interpretation. In: ACSM's guidelines for exercise testing and prescription, 6th edn. Philadelphia: Lippincott Williams \& Wilkins; 2000; $57-90$.

11. Goto Y, Itoh H, Adachi H, Ueshima K, Nohara R. Use of exercise cardiac rehabilitation after acute myocardial infarction: Comparison between health insurance-approved and non-approved hospitals in Japan. Circ J 2003; 67: 411 -415.

12. Goto Y, Sumida H, Ueshima K, Adachi H, Nohara R, Itoh H. Safety and implementation of exercise testing and training after coronary stenting in patients with acute myocardial infarction. Circ J 2002; 66: $930-936$.

13. Takagi S, Sakuragi S, Baba T, Takaki H, Aihara N, Yasumura Y, et al. Predictors of left ventricular remodeling in patients with acute myocardial infarction participating in cardiac rehabilitation: Brain natriuretic peptide and anterior infarction. Circ J 2004; 68: 214-219.

14. Koizumi T, Miyazaki A, Komiyama N, Sum K, Nakasato T, Masuda $\mathrm{Y}$, et al. Improvement of left ventricular dysfunction during exercise by walking in patients with successful percutaneous coronary intervention for acute myocardial infarcition. Circ J 2003; 67: 233-237.

15. Pashkow FJ, Dafoe WA. Clinical cardiac rehabilitation: A cardiologist's guide, 2nd edn. Philadelphia: Lippincott Williams \& Wilkins; $1999 ; 3-25$.

16. Taniguchi K, Itoh H, Yajima T, Doi M, Niwa A, Marumo F. Predischarge early exercise therapy in patients with acute myocardial infarction on the basis of anaerobic threshold (AT). Jpn Circ J 1990; 54: $1419-1425$.

17. American Association of Cardiovascular \& Pulmonary Rehabilitation. Cardiac rehabilitation in the inpatient and transitional settings. In: Guidelines for cardiac rehabilitation and secondary prevention programs, 3th edn. Williams, Champaign, IL, Human Kinetics; 1999; $15-52$.

18. Shephard RJ, Allen C, Benade AJ, Davies CT, Di Prampero PE, Hedman R, et al. Standardization of submaximal exercise tests. Bull World Health Organ 1968; 38: 765-775.

19. Wicks JR, Sutton JR, Oldridge NB, Jones NL. Comparison of the electrocardiographic changes induced by maximam exercise testing with treadmill and cycle ergometer. Circulation 1978; 57: $1066-$ 1070.

20. Miles DS, Critz JB, Knowlton RG. Cardiovascular, metabolic, and ventilatory responses of women to equivalent cycle ergometer and treadmill exercise. Med Sci Sports Exerc 1980; 12: 14-19.

21. Turley KR, Wilmore JH. Cardiovascular responses to treadmill and cycle ergometer exercise in children and adults. J Appl Physiol 1997; 83: $948-957$.

22. Vesovic D, Borjanovic S. Comparison of certain biochemical changes during exercise tests on treadmill and bicycle-ergometer with equal workload intensity. Med Lav 2001; 92: 130-136.

23. Senaratne MP, Smith G, Gulamhusein SS. Feasibility and safety of early exercise testing using the Bruce protocol after acute myocardial infarction. J Am Coll Cardiol 2000; 35: 1212-1220.

24. Gill TM, DiPietro L, Krumholz HM. Role of exercise stress testing and safety monitoring for older persons starting an exercise program. JAMA 2000; 284: 342-349.

25. Matsunaga A, Nakamura M, Masuda T, Izumi T. Adaptation to a cycle ergometer exercise in patients with acute myocardial infarction undergoing a Phase I cardiac rehabilitation. Kitasato Med 2000; 30: $439-449$.

26. Gamero LG, Vila J, Palacios F. Wavelet transform analysis of heart rate variability during myocardial ischaemia. Med Biol Eng Comput 2002; 40: $72-78$.

27. Verlinde D, Beckers F, Ramaekers D, Aubert AE. Wavelet decomposition analysis of heart rate variability in aerobic athletes. Auton Neurosci 2001; 90: 138-141.

28. Aubert AE, Ramaekers D, Beckers F, Breem R, Denef C, Van de Werf $\mathrm{F}$, et al. The analysis of heart rate variability in unrestrained rats: Validation of method and results. Comput Methods Programs Biomed 1999; 60: 197-213.

29. Spielberger CD, Gorsuch RL, Lushene RE. Manual for the state-trait anxiety inventory. PaloAlto: Consulting Psychologists Press, 1970.

30. Yoshida T, Yoshida K, Yamamoto C, Nagasaka M, Tadaura H, Meguro T, et al. Effects of a two-week, hospitalized phase II cardiac rehabilitation program on physical capacity, lipid profiles and psychological variables in patients with acute myocardial infarction. Jpn Circ J 2001; 65: 87-93.

31. Balady GJ, Fletcher BJ, Froelicher ES, Hartley LH, Krauss RM, Oberman A, et al. Cardiac rehabilitation programs: A statement for healthcare professionals from the American Heart Association. Circulation 1994; 90: $1602-1610$.

32. Oldridge NB, Jones NL. Preventive use of exercise rehabilitation after myocardial infarction. Acta Med Scand Suppl 1986; 711: $123-$ 129.

33. Evenson KR, Fleury J. Barriers to outpatient cardiac rehabilitation participation and adherence. J Cardiopulm Rehabil 2000; 20: 241 246.

34. Carlson JJ, Johnson JA, Franklin BA, VanderLaan RL. Program participation, exercise adherence, cardiovascular outcomes, and program cost of traditional versus modified cardiac rehabilitation. Am J Cardiol 2000; 86: 17-23.

35. American Association of Cardiovascular \& Pulmonary Rehabilitation. Special population. In: Guidelines for cardiac rehabilitaion and secondary prevention programs, 3th edn. Williams, Champaign, IL, Human Kinetics; 1999; 131-172.

36. Mark AL, Victor RG, Nerhed C, Wallin BG. Microneurographic studies of the mechanisms of sympathetic nerve responses to static 
exercise in humans. Circ Res 1985; 57: 461-469.

37. Victor RG, Seals DR, Mark AL. Differential control of heart rate and sympathetic nerve activity during dynamic exercise: Insight from intraneural recordings in humans. J Clin Invest 1987; 79: 508-516.

38. Seals DR, Chase PB, Taylor JA. Autonomic mediation of the pressor responses to isometric exercise in humans. J Appl Physiol 1988; 64: $2190-2196$

39. Saito M, Tsukanaka A, Yanagihara D, Mano T. Muscle sympathetic nerve responses to graded leg cycling. J Appl Physiol 1993; 75: $663-$ 667.

40. Bristow JD, Honour AJ, Pickering GW, Sleight P, Smyth HS Diminished baroreflex sensitivity in high blood pressure. Circulation 1969; 39: 48-54.

41. Eckberg DL, Drabinsky M, Braunwald E. Defective cardiac parasympathetic control in patients with heart disease. $N$ Engl J Med 1971; 285: 877-883.

42. Ryan C, Hollenberg M, Harvey DB, Gwynn R. Impaired parasympathetic responses in patients after myocardial infarction. Am J Cardiol 1976; 37: 1013-1018.
43. Saito M, Kagaya J. Human circulation: Regulation of oxygen transport system during exercise. Tokyo: NAP, 1999; 63-80.

44. Oya M, Itoh H, Kato K, Tanabe K, Murayama M. Effects of exercise training on the recovery of the autonomic nervous system and exercise capacity after acute myocardial infarction. Jpn Circ J 1999; 63: $843-848$

45. Bigger JT Jr, Fleiss JL, Rolnitzky LM, Steinman RC, Schneider WJ. Time course of recovery of heart period variability after myocardial infarction. J Am Coll Cardiol 1991; 18: 1643-1649.

46. Coats AJ, Adamopoulos S, Radaelli A, McCance A, Meyer TE Bernardi L, et al. Controlled trial of physical training in chronic hear failure: Exercise performance, hemodynamics, ventilation, and autonomic function. Circulation 1992; 85: 2119-2131.

47. Kiilavuori K, Toivonen L, Naveri H, Leinonen H. Reversal of autonomic derangements by physical training in chronic heart failure assessed by heart rate variability. Eur Heart J 1995; 16: 490-495.

48. Bortz WM. Disuse and aging. JAMA 1982; 248: 1203-1208.

49. Robergs RA, Roberts SO. Exercise and aging: Exercise physiology. Maryland: Mosby; 1997; 578-599. 